\section{Diversité Et Distribution De La Grande Faune Mammalienne Dans Le Parc National De Moukalaba-Doudou (Sud-Ouest Du Gabon)}

\section{Fred Loïque Mindonga Nguelet,} Département de Biologie et Ecologie Animale, Institut de Recherche en Ecologie Tropicale (IRET)/ CENAREST, , Libreville, Gabon

\section{Lilian Brice Mangama-Koumba, Faculté des Sciences et Techniques (FAST)/} Université d’Abomey-Calavi, Cotonou, Benin.

\section{Ghislain Wilfried Ebang Ella,} Département de Biologie et Ecologie Animale, Institut de Recherche en Ecologie Tropicale (IRET)/ CENAREST, Libreville, Gabon

\section{Aubin Armel Koumba,}

Département de Biologie et Ecologie Animale, Institut de Recherche en Ecologie Tropicale (IRET)/ CENAREST, , Libreville, Gabon Faculté des Sciences et Techniques (FAST)/ Université d’Abomey-Calavi, Cotonou, Benin

\section{Jacques François Mavoungou,}

Département de Biologie et Ecologie Animale, Institut de Recherche en Ecologie Tropicale (IRET)/ CENAREST, , Libreville, Gabon

Submitted: 20 February 2020

Accepted: 23 July 2020

Published: 31 December 2020

Corresponding author:

Mangama Koumba Lilian Brice

DOI: $\underline{10.19044 / \text { esj.2020.v16n36p34 }}$ Distributed under Creative Commons BY-NC-ND 4.0 OPEN ACCES

\section{Cite as:}

Mangama-Koumba L, Ebang Ella G, Koumba A., Mavoungou J. (2020). Diversité et distribution de la grande faune mammalienne dans le Parc National de Moukalaba-Doudou (Sud-Ouest du Gabon). European Scientific Journal, ESJ, 16 (36), 1. https://doi.org/10.19044/esj.2020.v16n36p34

\section{Résumé \\ Le Gabon, pays d'Afrique Centrale est} recouvert à plus de $80 \%$ de la forêt pourvue des zones diversifiées en paysages avec une importante biodiversité faunique et floristique. Cependant, cette biodiversité qui est mal connue dans certaines régions du pays est confrontée à plusieurs menaces. C'est dans cette optique que nous avons conduit un suivi de la faune dans la partie Nord Est du parc national de Moukalaba Doudou dont le but était de déterminer la diversité et la répartition spatiale des grands mammifères sauvages. En utilisant la méthode de line transect, nous avons installé 165 cameras traps le long de 11 transects. Les données ont été performées par le GML (Generalized Linear Models). $\mathrm{Au}$ total, 80410 nuits-pièges ont été réalisées. Près de 37914 vidéos indépendantes ont été obtenues et 10 espèces de grands mammifères dont les plus observés étaient les Céphalophes bleus et rouges suivis des Chevrotains aquatiques, Eléphants et des Cercocèbes. Les résultats obtenus suggèrent que les espèces de grands mammifères sont distribuées en fonction des habitats. Les céphalophes de grande taille, le mandrill, la panthère, le chat doré, l'éléphant, et le chevrotain aquatique ont des habitats préférentiels ; par contre, le gorille, le chimpanzé, le cercocèbe à collier blanc et le céphalophe bleu ne semblent pas être affectés par l'habitat. Ce sont des espèces ubiquistes. Cependant, tous les mammifères peuvent être observés sur l'ensemble des transects.

Subject: Biology 
European Scientific Journal, ESJ

December 2020 edition Vol.16, No.36
ISSN: 1857-7881 (Print) e - ISSN 1857-7431

Mots-clés: Biodiversity, Mammal, Distribution, Camera trap And Habitat 


\section{Diversity And Distribution Of Large Mammalian Fauna In Moukalaba-Doudou National Park (South- Western Of Gabon)}

\section{Fred Loïque Mindonga Nguelet,}

Département de Biologie et Ecologie Animale, Institut de Recherche en

Ecologie Tropicale (IRET)/ CENAREST,Libreville, Gabon

\section{Lilian Brice Mangama-Koumba,}

Faculté des Sciences et Techniques (FAST)/ Université d'Abomey-Calavi, Cotonou, Benin.

\section{Ghislain Wilfried Ebang Ella,}

Département de Biologie et Ecologie Animale, Institut de Recherche en

Ecologie Tropicale (IRET)/ CENAREST, Libreville, Gabon

\section{Aubin Armel Koumba,}

Département de Biologie et Ecologie Animale, Institut de Recherche en Ecologie Tropicale (IRET)/ CENAREST, Libreville, Gabon Faculté des Sciences et Techniques (FAST)/ Université d'Abomey-Calavi, Cotonou, Benin

Abstract

Gabon, a country in Central Africa, is covered by more than $80 \%$ of the forest, with areas diversified into landscapes with significant fauna and flora biodiversity. However, this biodiversity, which is poorly understood in certain regions of the country, faces several threats. It is with this in mind that we conducted wildlife monitoring in the northeast part of Moukalaba Doudou National Park, the aim of which was to determine the abundance and spatial distribution of large wild mammals. Using the line transect method we installed 165 cameras traps along 11 transects. The data were performed by the GML (Generalized Linear Models). A total of 80,410 trap nights were completed. Almost 37,914 independent videos were obtained and 10 species of large mammals of which the most observed were the blue and red duikers followed by aquatic Chevrotains, Elephants and Mangabeys. The results obtained suggest that large mammal species are distributed according to habitats. Large duikers, mandrill, panther, golden cat, elephant, and aquatic chevrotain have preferred habitats; however, the gorilla, the chimpanzee, the white-collared cercocebe and the blue duiker do not seem to be affected by the 
habitat. They are ubiquitous species. However, all mammals can be seen on all of the transects.

Keywords: Biodiversity, Mammal, Distribution, Camera Trap And Habitat

\section{Introduction}

Les forêts africaines couvrent une superficie de 635412 hectares et représentent 16\% des forêts du monde (Angoni Avom et Ngo, 2013). Parmi elles, on y trouve les forêts du bassin du Congo qui constituent la plus grande zone de forêts tropicales au monde après l'Amazonie (Rieucau, 2001). Ces forêts regorgent une biodiversité riche en espèces animales et végétales. En effet, les forêts du bassin du Congo abritent une diversité biologique estimée à 400 espèces de mammifères, 1300 espèces d'oiseaux, 336 espèces d'amphibiens, 400 espèces de reptiles et 20000 espèces de plantes parmi lesquelles 800 espèces sont endémiques (Fern et al., 2008).

Au Gabon, pays du bassin du Congo, 88\% du territoire national (23,6 millions d'ha) se trouve recouvert par une forêt très fortement diversifiée en paysages avec une importante diversité d'espèces végétales et animales (Ernst et al., 2012) dont 150 espèces de mammifères, 600 espèces d'oiseaux, 100 espèces d'amphibiens et près de 8000 espèces de plantes (CBD, 2013). Cependant, cette importante biodiversité est généralement menacée par la déforestation, l'agro-industrie, les usages du sol et les pratiques de gestion, auxquelles s'ajoutent les changements climatiques, le trafic de la viande de brousse, etc. (Roger et al., 2006 ; Mambeya et al., 2018).

Pour faire face à ces menaces et dans un souci de préserver cet important patrimoine naturel, une ambitieuse politique de conservation a été mise en place en 2002, conduisant à la création de 13 Parcs Nationaux qui couvrent 11\% du territoire (soit 3013842 d'hectares) (Doumenge et al., 2015).

Parmi ces aires protégées se trouve le Parc National de Moukalaba Doudou (PNMD), le troisième Parc National du Gabon en termes de superficie (449 548 ha). Il est caractérisé par sa grande diversité d'habitats avec une mosaïque forêt-savane, et surtout, par sa forte densité en grands singes (gorilles et chimpanzés) (Takenoshita et al.,2008), en céphalophes (Nakashima et al., 2013) et en petits singes (Mangama et al., 2016). De plus, le PNMD abrite de nombreuses espèces de grands mammifères (Mindonga Nguelet et al., 2016).

Les connaissances sur la distribution de cette faune dans le parc national de Moukalaba-Doudou restent encore insuffisantes et fragmentaires Car seule une étude à long terme sur les gorilles des plaines de l'Ouest reste d'actualité. Pourtant, il est actuellement admis que la gestion durable des ressources naturelles d'un espace dépend non seulement d'une meilleure 
connaissance de la diversité de ses éléments constitutifs, mais également de leurs abondances (Evans et al., 2018).

L'objectif de cette étude était d'inventorier et de mieux connaitre la répartition spatiale des grands mammifères sauvages dans le PNMD.

\section{Matériel et méthodes}

\section{1- Zone d'étude}

La présente étude a été réalisée au Nord-Est du Parc National de Moukalaba-Doudou (PNMD), dans un site qui s'étend sur près de $30 \mathrm{~km}^{2}$ (Ando et al., 2008) (Figure 1). Cette aire protégée qui couvre une superficie de $5028 \mathrm{~km}^{2}$, est localisé dans la partie sud-ouest du Gabon, à $700 \mathrm{~km}$ de Libreville (Iwata et Ando, 2007 ; Ando et al., 2008 ; Takenoshita et Yamagiwa, 2008).

La végétation, au sein de ce parc est globalement subdivisé en quatre types, à savoir : les vieilles forêts secondaires (Pycnantus guineensis et Nauclea didenrichii), les forêts inondables (Uapaca guineensis), les jeunes forêts secondaires (à Musanga cecropioides et Aframomum sp.) et les savanes (Hyparrenhia diplandra et Panicum phragmithoide) (Iwata et Ando, 2008 ; Takenoshita et al., 2008). Les trois premiers habitats sont colonisés par de nombreuses espèces végétales dont les plus abondantes sont Musanga cecropoides, Aframomum sp, Cissus dinklagie, Myrianthus arboreus, Irvingia gabonensis, Dichostemma glaucescens, Meiocarpidium lepidetum et Diospyros sp. (Mounioko et al., 2016). La présence de ces plantes témoigne d'une forêt déjà perturbée par l'activité humaine, notamment l'exploitation forestière, l'agriculture. (ANPN, 2016).

Le paysage est caractérisé par une diversité d'habitats (plages et dunes côtières, mangroves, forêts littorales, marais d'eau douce et saumâtres, forêt de terrain bas inondée de façon saisonnière ou permanente, forêt de terrain élevé, surfaces rocheuses, forêts secondaires à différents stades, forêts primaires, prairies, marais à papyrus et à Raphia, savane, et vastes marécages doux et saumâtres) qui rend ce parc attractif pour les activités écotouristiques et de recherche (Vande Weghe, 2012).

Son climat est de type équatorial, caractérisé par une saison des pluies d'Octobre à Avril et une saison sèche de Mai à Septembre. Les précipitations annuelles varient entre $1582 \mathrm{~mm}$ et $1886 \mathrm{~mm}$. Par contre, les températures minimales et maximales varient respectivement entre 21,3 et $24,1^{\circ} \mathrm{C}$, puis 29,3 et $33,7^{\circ} \mathrm{C}$ (Takenoshita et Yamagiwa, 2008).

Le PNMD abrite un relief plat vers la côte, légèrement ondulé vers l'intérieur des terres. Dans ces endroits, des collines escarpées dont l'altitude varie entre 600 et $800 \mathrm{~m}$ se développent dans les Monts Doudou (Campbell et al., 2006 ; ANPN, 2016). 


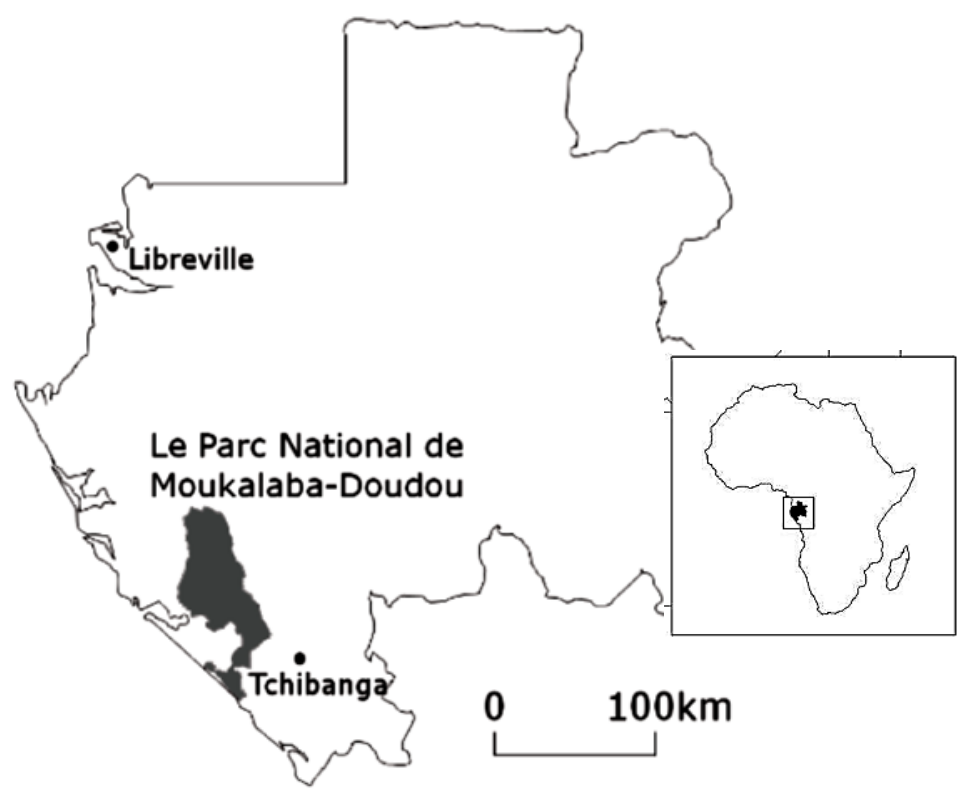

Figure 1 : Localisation géographique de la zone d’étude (Mindonga Nguelet, 2017)

\section{2- Inventaire des grands mammifères}

La collecte des données sur les grands mammifères de notre zone d'étude a été faite de janvier 2012 à janvier 2014 en utilisant 10 caméras pièges par transect équidistants de $200 \mathrm{~m}$. L'effort total de capture était de 165 caméras pièges (Cam Bushnell Trophy), soit un total de 10 cameras par transects. En pratique, nous avons disposé ces caméras pièges le long de 11 transects de $2 \mathrm{~km}$, à savoir 2 transects dans les jeunes forêts secondaires (A et $\mathrm{KO}), 2$ transects dans la forêt marécageuse (G22, BV), 6 transects dans les vieilles forêts secondaires (MB, DT, NK, DB, G5, FD) et 1 transect dans la forêt primaire et des montagnes (MD).

Chaque caméra piège a été attachée à un arbre, à $10 \mathrm{~m}$ de la ligne des transects, à $30 \mathrm{~cm}$ du sol (Nakashima, 2015; Hongo et al., 2016). Par ailleurs, l'emplacement de toutes les caméras pièges a été géoréférencé en utilisant un GPS MAP 60 CSX de marque GARMIN. Ces cameras pièges ont été visitées une fois par mois durant la période d'étude afin de changer les batteries et les cartes mémoires SD. 


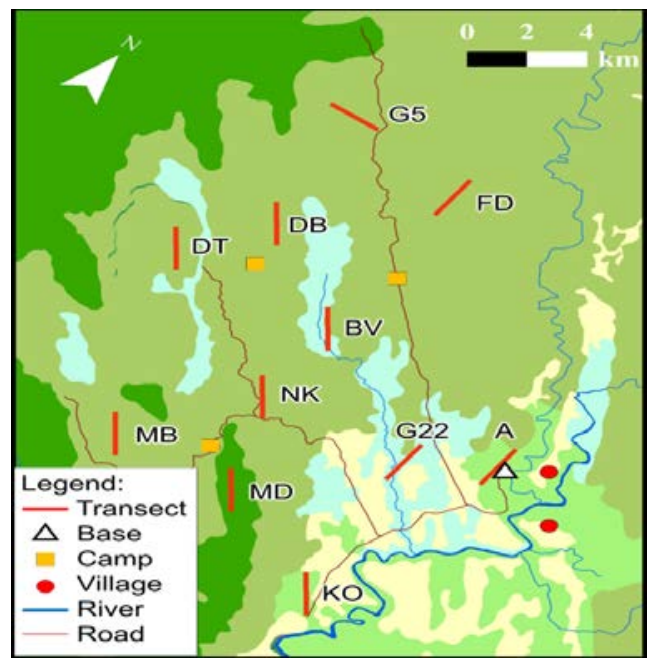

Figure 2 : Carte schématique de la zone d'étude dans la partie Est-centrale du Parc National de Moukalaba Doudou (Gabon) montrant les différents transects et habitats

\section{3- Visualisation et identification des mammifères}

Au cours de cette étude, les caméras pièges ont été mises en mode vidéo afin de permettre la capture d'images vidéo à des séquences très courtes (Kierulff et al., 2004). Les images vidéo ont été enregistrées à 30 secondes. Chaque image vidéo a été considérée comme étant la preuve d'une seule visite par une espèce donnée, dans le cas où l'image a été obtenue à un intervalle supérieur à 30 minutes après l'image précédente de la même espèce (O’brien et al., 2003 ; Nakashima, 2015). Chaque image vidéo acquise a été enregistrée avec la date et l'heure de la prise de vue. Par contre, les images vidéo ne comportant pas ces informations ont été exclues de l'analyse des données.

En outre, les données obtenues ont été filtrées pour exclure les images vidéo de la même espèce acquises avec la même caméra piège, dans un délai de $30 \mathrm{~min}$. Cela permet d'assurer l'indépendance de chaque capture, car certaines espèces (par exemple, l'éléphant, les céphalophes, le gorille, le chimpanzé) passent beaucoup de temps devant une caméra piège (Nakashima et al, 2013).

Tous les grands mammifères filmés par les caméras pièges, ont été visualisés puis identifiés selon la clé de détermination de Dorst et Dandelot (1993) puis celle de Kingdon et al. (2013). Une attention particulière a été portée sur les espèces animales comme le chimpanzé (Pan troglodytes troglodytes), le gorille (Gorilla gorilla), le mandrill (Mandrillus sphinx), cercocèbe à collier blanc (Cerccocebus torquatus torquatus), l'éléphants (Loxodonta africana cyclotis), le potamochère (Potamochoerus porcus), le chevrotain aquatique (Hyemoschus aquaticus), le céphalophe bleu (Cephalophus monticola), le céphalophe à dos jaune (Cephalophus sivicultor), 
les céphalophes rouges (céphalophe d'Ogilby, céphalophe de peters et céphalophe bai), la panthère (Panthera perdus) et le chat doré (Felis aurata). Ces animaux ont été ciblés car ils seraient les plus abondants parmi les grands mammifères du PNMD et ont pour certaines espèces une priorité de conservation élevée au Gabon.

Par ailleurs, il est à noter que les petits et grands rongeurs, les petits carnivores, les ongulés herbivores savanicoles (cobe, buffle et sitatunga), certaines espèces arboricoles et les pangolins n'ont pas été pris en compte dans cette étude, à cause de leur très faible taux de capture et de la faible valeur de conservation de certaines de ces espèces animals

\subsection{Analyse statistique des données}

Les données de terrain ont été saisies et organisées dans le tableur Excel 2013. Pour déterminer l'abondance relative de la grande faune mammalienne, nous avons calculé le taux de capture de chaque espèce qui correspond au nombre d'images vidéo/1000 jours nuits-piège (Tobler et al., 2008 ; Nakashima, 2013).

Par ailleurs, nous avons utilisé le modèle linéaire généralisé GLM (Generalized Linear Models) afin de mieux appréhender la distribution spatiale de ces animaux. Ces modèles ont été équipés d'une distribution gaussienne d'erreur et de fonction de lien linéaire. A cet effet, les paramètres tels que la savane, les marais, l'erreur moyenne et l'erreur standard ont été utilisés comme la potentielle co-variable. Au début, toutes les quatre variables ont été incluses au modèle complet. Par la suite, ce modèle a été simplifié à partir de la fonction StepAIC

\section{2- Résultats}

\section{1- Richesse et composition spécifique}

Au total, 80410 nuits-pièges ont été réalisées dans le cadre de cette étude. Près de 38823 vidéos indépendantes ont été obtenues (événements indépendants ne tenant pas compte des photos réalisées à quelques minutes d'intervalle).

Douze (12) espèces de mammifères a été observé dans la zone d'étude. Le taxon le plus riche est le genre Cephalophus avec 3 espèces. Par contre, le reste des genres a été représenté par une seule espèce (Tableau 1).

Tableau 1 : Genre de grands mammifères observés dans le PNMD

\begin{tabular}{ll}
\hline Genre & Nombre d'espèces \\
\hline Pan & 1 \\
Gorilla & 1 \\
Mandrillus & 1 \\
Cercocebus & 1 \\
\hline
\end{tabular}




\begin{tabular}{ll}
\hline Loxodonta & 1 \\
\hline Potamochoerus & 1 \\
Hyemoschus & 1 \\
Cephalophus & 3 \\
\hline Panthera & 1 \\
Felis & 1 \\
\hline
\end{tabular}

\section{2- Taux de capture des mammifères rencontrés au PNMD}

L'analyse des résultats du tableau 2 fait ressortir que le céphalophe bleu, les céphalophes rouges, le chevrotain aquatique, l'éléphant et le Cercocèbe à collier blanc sont les mammifères les plus observer dans la zone d'étude avec des taux de capture de 201,$7 ; 134,4 ; 28,7$; 28,5 et 20,9 respectivement. Les autres espèces notamment le chimpanzé, le gorille, le mandrill, potamochère, le céphalophe à dos jaune, la panthère et le chat doré ont été faiblement représentés avec un taux de capture $\leq 20$.

Tableau 2: Taux de capture des grands mammifères dans le PNMD au Gabon.

\begin{tabular}{lllc}
\hline Ordre & Nom scientifique & Nom commun & Taux de capture (n) \\
\hline Primates & Pan troglodytes & Chimpanzé & $9,8(784)$ \\
& Gorilla gorilla & Gorille & $10(804)$ \\
& Mandrillus sphinx & Mandrill & $6,7(538)$ \\
& Cercocebus torquatus & Cercocèbe à collier blanc & $20,9(1678)$ \\
\hline Proboscidae & Loxodonta africana & Eléphant & $28,5(2289)$ \\
\hline Certartiodactyla & Potamochoerus porcus & Potamochère & $11,9(960)$ \\
& Hyemoschus aquaticus & Chevrotain aquatique & $28,7(2310)$ \\
& Cephalophus monticola & Céphalophe bleu & $201,7(16218)$ \\
& Cephalophus silvicultor & Céphalophe à dos jaune & $18,9(1523)$ \\
& Cephalophus spp & Céphalophes rouges & $134,4(10810)$ \\
\hline Carnivores & Panthera pardus & Léopard & $6,23(501)$ \\
& Felis aurata & Chat doré & $5,07(408)$ \\
\hline
\end{tabular}

\section{3- Distribution spatiale des grands mammifères}

Les espèces de grands mammifères sont distribuées en fonction des habitats. Les céphalophes rouges, le céphalophe à dos jaune, le mandrill, la panthère, le chat doré, l'éléphant, et le chevrotain aquatique ont des habitats préférentiels. Par contre le gorille, le chimpanzé, le cercocèbe à collier blanc et le céphalophe bleu ne semblent pas être affectés par l'habitat (figure 3): Ce sont des espèces ubiquistes. Tous les grands mammifères peuvent être observables sur l'ensemble des transects linéaires (figure 3). 
La distribution des grands mammifères inventoriés sur l'ensemble des 11 transects, variait en fonction de l'habitat (type de végétation) et de la distance des villages (figure 3). La figure 3 montre que parmi les primates, seuls le mandrill et le chimpanzé ont été les moins capturés dans les jeunes forêts secondaires proches des villages, suivi des grands carnivores, (panthère et chat doré), et des ongulés (3 céphalophes rouges, le potamochère). Le cercocèbe à collier blanc est moins abondant dans les zones de haute altitude, suivi de l'éléphant, du céphalophe bleu et des 3 céphalophes rouges. Le chimpanzé, le cercocèbe à collier blanc, les céphalophes rouges et le potamochère sont beaucoup plus abondants dans les vieilles forêts secondaires.
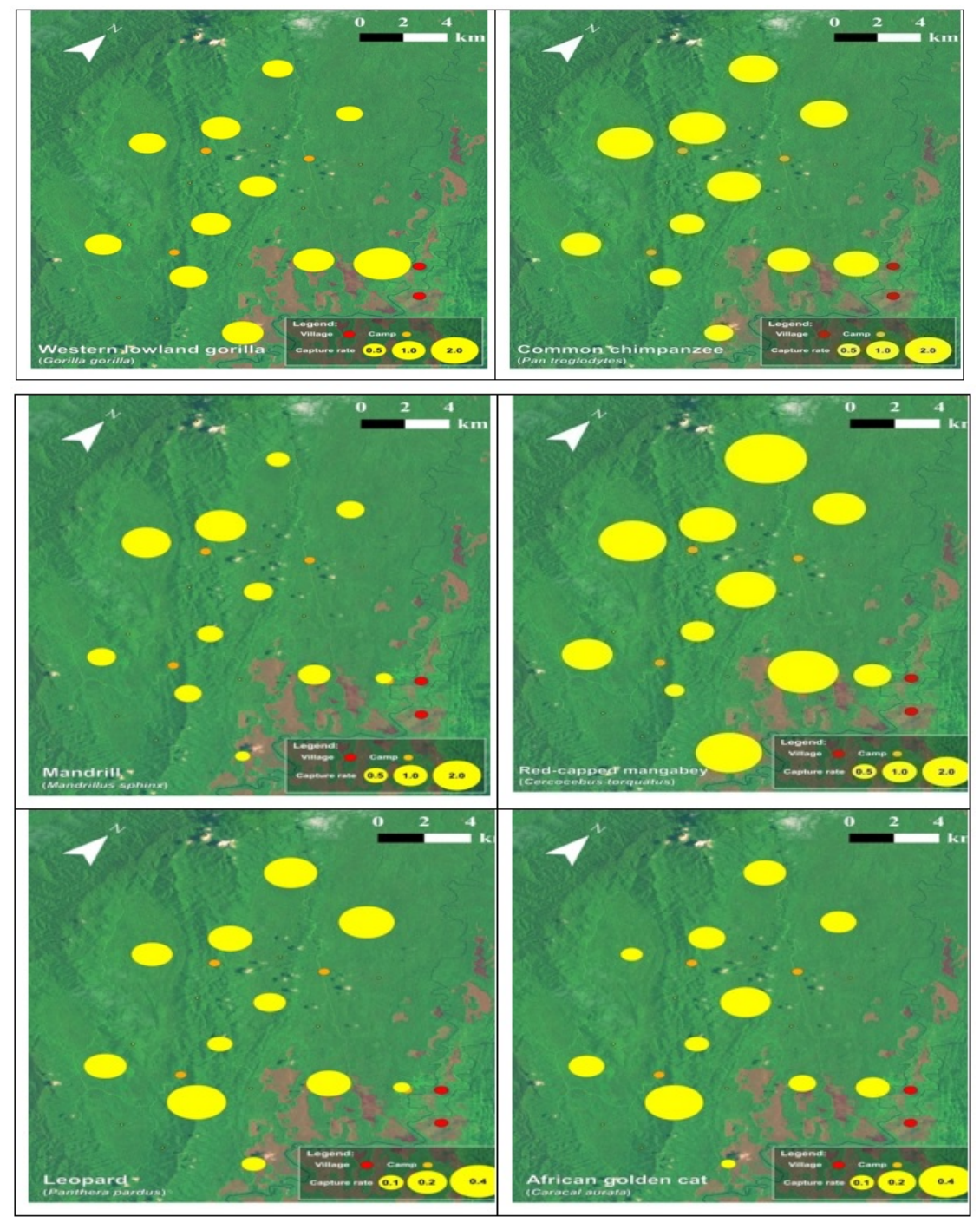


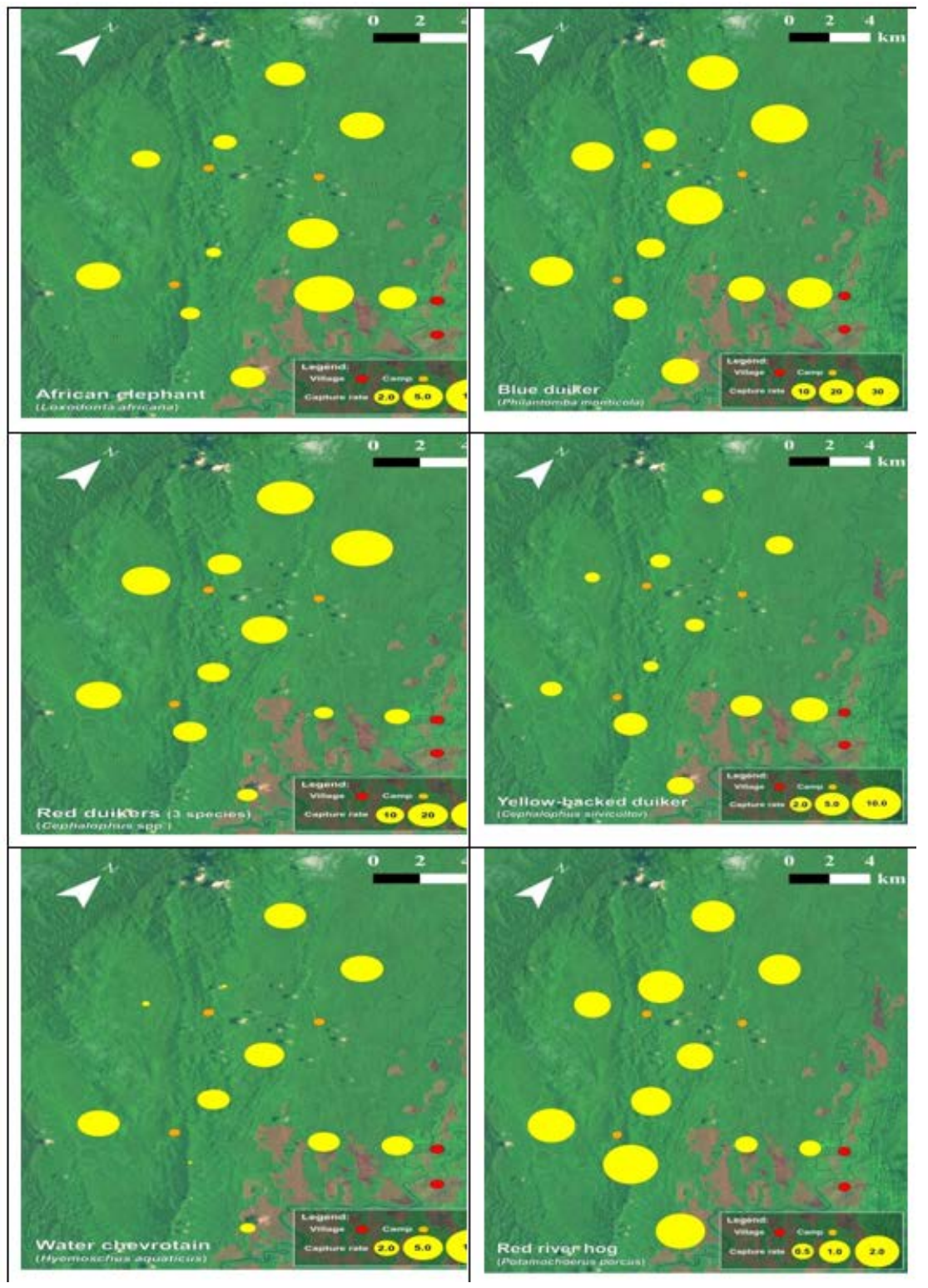

Figure 3: Carte de distribution des grands mammifères dans la zone d'étude.

\section{3- Discussion}

Les résultats de cette étude donnent une information supplémentaire sur la diversité des grands mammifères sauvages dans la partie nord du PNMD. L'étude qualitative de la grande faune mammalienne de la zone d'étude a montré une bonne richesse spécifique avec la présence de 10 espèces composées essentiellement d'herbivores et de primates. Toutes ces espèces ont un intérêt de conservation en Afrique centrale (Binot, 2010). 
L'usage des caméras pièges dans cette étude a été très utile puisqu'elle a permis de mettre en relief via des vidéos, la présence d'espèces difficiles à voir en temps normal, à l'image des chimpanzés et des céphalophes (Cibot et al, soumis). D’ailleurs, Burton et al (2015) ont signalé que les caméras pièges détectent un nombre d'espèces 1,5 fois supérieur à celui des observations directes. Ces résultats corroborent ceux de Pebsworth et Lafleur (2014). Ces auteurs pensent que la méthode des pièges photographiques est très utile pour l'étude des espèces de mammifères rares ou nocturnes et permet d'obtenir des informations assez satisfaisantes (Trolliet et al., 2014).

Cependant, la faiblesse du nombre d'espèces enregistrées pourrait être liée à l'utilisation d'une seule méthode d'inventaire des grands mammifères. En effet, Bouché (2008) préconise l'utilisation de plusieurs méthodes d'échantillonnage lors des inventaires plurispécifiques afin de maximiser la diversité des collectes.

En outre, les résultats sur la distribution et l'abondance des espèces de grands mammifères ont montré que leur coexistence est généralement expliquée par la disponibilité de la ressource alimentaire (Gauthier-Hion et al., 1985), mais aussi par la nature de l'habitat. En effet, l'ensemble des espèces de grands mammifères étudié sont distribuées de façon homogène. Leur abondance varie en fonction de l'habitat et de l'espèce. Dans cette présente étude, Cephalophus monticola, Cephalophus spp., Hyemoschus aquaticus, Loxodonta africana cyclotis et Cercocebus torquatus torquatus sont les espèces les plus abondantes sur l'ensemble de notre site d'étude.

Les gorilles sont plus distribués et abondants aux abords des villages. Effectivement, les forêts proches du village ont été longtemps exploitées par les villageois et ces forêts fournissent une variété des ressources alimentaires aux gorilles. Dans d'autres sites, notamment en République Démocratique du Congo (RDC), Yamagiwa et Basabose (2006) avaient observé le même résultat où les gorilles préféraient côtoyer les villages car les herbes terrestres leur fournissaient une multitude de nourriture. Par contre, les chimpanzés, les mandrills et les cercocèbes à collier blanc sont distribués et abondants dans les vieilles forêts secondaires et primaires. En République Démocratique du Congo (RDC), Basabose (2002) avait montré le même résultat, où les chimpanzés préféraient les vieilles forêts secondaires et les forêts primaires qui offraient beaucoup plus les fruits. Au Gabon, notamment au Parc National de Moukalaba Doudou les résultats de Mangama et al. (2016) corroborent les nôtres, montrant que les petits singes préfèrent également les vieilles forêts secondaires et les forêts primaires car, ils ont une préférence sur la consommation les fruits. A la Lopé, Tutin et Fernandez (1993) avaient aussi montré que les petits primates ont une alimentation dominée par les fruits et les graines. Des études antérieures ont suggéré que les gorilles et les chimpanzés ont des préférences soit alimentaire, soit au niveau du degré de 
perturbation de la forêt. Les chimpanzés sont en effet très vulnérables à l'exploitation forestière car ils sont territoriaux (White et Edwards, 2002). D'autre part, les gorilles préfèrent les forêts secondaires qui fournissent une plus grande quantité de ressources alimentaires (Tutin et Fernandez, 1984).

Par ailleurs, les résultats de cette étude suggèrent que les céphalophes, le potamochère et le chevrotain aquatique n'ont pas la même occupation des transects. Les céphalophes rouges (Cephalophus sp.), le céphalophe bleu (Cephalophus monticola), le chevrotain aquatique (Hyemoschus aquaticus) et le potamochère (Potamochoerus porcus) sont moins distribués dans les jeunes forêts secondaires c'est-à-dire le long des transects proches des villages. Le céphalophe à dos jaune (Cephalophus sylvicultor) ne l'est pas. Dans le Parc National de Moukalaba-Doudou, des études antérieures avaient suggéré que les céphalophes rouges étaient abondants dans les vieilles forêts secondaires et les forêts primaires, mais très rares dans les jeunes forêts secondaires et les galeries forestières (Nakashima et al., 2013). De plus, Akomo et al. (2015) ont montré que l'abondance de crottes des céphalophes rouges, céphalophe bleu et le chevrotain aquatique étaient affectés par les perturbations humaines, tandis que le céphalophe à dos jaune ne l'était pas. Aussi, l'abondance de crottes des céphalophes rouges était plus faible dans les jeunes forêts secondaires qui se régénèrent après une importante perturbation humaine. En revanche, l'abondance de crotte de céphalophe bleu n'était pas affectée par le type de végétation. Elle était plus faible dans les transects proches des villages, ce qui pourrait être dû à l'activité de la chasse dans le passé (Akomo et al., 2015). La distribution et l'abondance du potamochère et du chevrotain aquatique dépendent également du type de végétation. Ces dernières préfèrent fréquenter les végétations denses qui leur procurent les fruits, graines et racines, mais aussi de terriers pour se cacher des prédateurs. Tutin et al. (1997) ont montré à la Lopé que le potamochère et le chevrotain aquatique aimaient fréquenter beaucoup plus les forêts primaires et les forêts marécageuses car la majeure partie de leur régime alimentaire est constituée des fruits (potamochère et chevrotain aquatique), graines, racines et vers de terre (potamochère). En outre, la distribution et l'abondance des grands carnivores (la panthère et le chat doré) dans les jeunes forêts secondaires pourrait s'expliquer du fait que leurs proies favorites notamment, le potamochère, les céphalophes et les petits primates ont une très faible abondance dans les transects proches des villages. Concernant les éléphants, ils sont distribués et présents dans l'ensemble de notre zone d'étude. Mais ils préfèrent aussi fréquenter les transects proches des villages. Car, ils ont augmenté le contact avec les humains en raison de changements dans l'utilisation des terres qui ont été converties en culture (Nelson et al. 2003). C'est ce qui est à l'origine des conflits homme/faune. Le même résultat a été observé à Makokou (Barnes et al., 1995) et à la Lopé (White, 1994; Tutin et al., 1997). 


\section{Conclusion}

Cette étude a permis de connaitre les espèces de la grande faune et leur distribution géographique. Elle donne un aperçu global des espèces de grands mammifères sauvages colonisant la zone nord du PNMD. De plus, cette étude révèle que les biotopes du PNMD hébergent une grande faune mammalienne relativement diversifiée. Les images des caméras pièges ont permis de confirmer la présence de 10 espèces de grands mammifères avec une prédominance des herbivores. Ainsi, le Parc National de Moukalaba-Doudou constitue un site approprié pour l'observation et la vision des grands mammifères. Cependant, la plupart de ces espèces sont vulnérables et menacées. Il serait intéressant de mener des études supplémentaires en combinant d'autres méthodes d'inventaires afin d'avoir une connaissance plus large des mammifères présents dans les habitats de cette aire protégée. Aussi, les résultats de cette étude constituent une base de données de référence pour des études scientifiques ou écotouristiques de la zone de Moukalaba Doudou.

\section{Remerciement}

Cette étude a été réalisée en coopération entre les Centre National de la Recherche Scientifique et Technologique (CENAREST), l'Institut de Recherche en Écologie Tropicale (IRET), l’Université des Sciences et Techniques de Masuku (USTM), Gabon et Kyoto Université, Japon. Nous remercions l'Agence Nationale des Parcs Nationaux du gouvernement gabonais pour autorisation et soutien pour notre projet de recherche au Gabon. Nous sommes également très redevables à tous les assistants de terrain du Parc National de Moukalaba-Doudou et les habitants des villages de Doussala, Konzi et Mboungou pour leur collaboration et leur hospitalité. Cette étude a été réalisée grâce au financement du programme de cours de doctorat de l'Université des Sciences et Techniques de Masuku (à F.L MINDONGA NGUELET) et aux subventions d'aide à la recherche scientifique du Ministère de l'Education, la Culture, des Sports, de la Science et de la Technologie du Japon (n ${ }^{\circ} 162550080, n^{\circ} 19107007, n{ }^{\circ} 24255010$ à J. Yamagiwa) et de SATREPS (Science et technologie Partenariat de recherche pour le développement durable) à travers JST / JICA (Japon).

\section{References:}

1. Akomo-Okoue, Ef.; Inoue, E.; Atteke, C.; Nakashima, Y.; Hongo, S.; Inoue-Murayama, M.; Yamagiwa, J. (2015). Non-invasive genetic analysis for assessing the abundance of duiker species among habitats in the tropical forest of Moukalaba, Gabon. Mammal Research, Electronic supplementary material, The online version of this article DOI 10.1007/s13364-015-0233-1. 
2. Ando, C.; Iwata, Y.; Yamagiwa, J. (2008). Progress of habituation of western lowland gorillas and their reaction to observers in MoukalabaDoudou National Park, Gabon. African Study Monographs Supplementary Issue, vol. 39: 55-69.

3. ANGONI AVON, CT,; NGO SAMNICK, EL. (2013). Ecotourisme dans le Bassin du Congo. Quel potentiel pour le développement durable des pays ? Editions universitaires européennes, Sarrebruck, Allemagne, 43p.

4. ANPN, 2016. Cadre de gestion environnementale et sociale (CGES). Rapport du projet de gestion de la faune et des conflits hommeéléphant dans le sud du Gabon, 176p.

5. BARNES, RFW,; BLOM, A,; ALERS, MPT,; BARNES, KL. (1995). An estimate of the numbers of forest elephants in Gabon. Journal of Tropical Ecology, 11:27-37.

6. BASABOSE, AK. (2002) Diet composition of chimpanzees inhabiting the montane forest of Kahuzi, Democratic Republic of Congo. American Journal of Primatology 58:1-21.

7. BOUCHE P, 2008. Méthodes d'inventaire de la Grande Faune à l'usage des ZCV. Méthodes d'inventaires 164p.

8. BURTON, A.C., NEILSON, E., MOREIRA, D., LADLE, A., STEENWEG, R., FISHER, J.T. et al. (2015). Wildlife camera trapping: a review and recommendations for linking surveys to ecological processes. Journal of Applied Ecology, 52: 675-685.

9. CAMPBELL, P,; RIVERA, P,; THAMAS, D,; BOUROBOUBOUROBOU, H,; NZABI, T,; ALONSO, A,; DALLMEIER, F. (2006). Structure, composition et diversité floristiques d'une forêt équatoriale du Gabon. Bulletin of the Biological Society of Washington, $\mathrm{n}^{\circ} 12: 29-51$.

10. CIBOT, M,; BORTOLAMIOL, S,; SEGUYA, A,; KRIEF, S (SOUMIS) Chimpanzees facing a dangerous situation: a high traffic alsphated road in sebitoli, kibale national park, Uganda.

11. DORST, J,; DANDELOT, P. (1993). Guide des mammifères d'Afrique. Delachaux et Nestlé, Lausanne.

12. DOUMENGE, C,; PALLA, F,; SCHOLTE, P,; HIOL-HIOL, F; LARZILLIERE, A (Eds.), (2015). Aires protégées d'Afrique centrale - État 2015. OFAC, Kinshasa, République Démocratique du Congo et Yaoundé, Cameroun, 256 p.

13. ERNST, C, ; VERHEGGHEN, A,; MAYAUX, P,; HANSEN, M; DEFOURNY, P,; BARAWANDIKA, A,; BEGOTO, G,; ESSONO MBA, F,; IBARA, M,; KONDJO SHORO, A. (2012) Cartographie du couvert forestier et des changements forestiers en Afrique Centrale. In l'état des forêts 2010. C. Wasseige, P. Marcken, N. Bayol, F. Hiol- 
Hiol, P. Mayaux, B. Desclée, R. Nasi, A. Billand, P. Defourny et R. Eba'a Atyl. Eds (Luxembourg : office des publications de l'Union Europeenne). Pp23-42.

14. EVANS, K,; GUARIGUATA, MR,; BRANCALION, PHS, (2018). Participatory monitoring to connect local and global priorities for forest restoration. Conservation Biology, 0, 0, 1-10.

15. FERN, GREENPEACE, INTER- AFRICAN FOREST INDUSTRY ASSOCIATION, PREVIOUS WOODS, SWEDISH. SOCIETY FOR NATURE CONSERVATION AND TROPICAL FOREST TRUST. (2008) Regaining credibility and rebuilding support: changes FSC needs to make to regain its credibility. Pp

16. GAUTIER-HION, A,; DUPLANTIER, JM,; QURIS, R,; FEER, F,; SOUND, C,; DECOUX, JP,; DUBOST, G,; EMMONS, L,; ERAND, C,; HECKETSWEILER, P,; MOUNGAZI, A,; ROUSSILHON, C,; THIOLLAY, JM. (1985). Fruit characters as a basis of fruit choice and seed dispersal in a tropical forest vertebrate community. Oecologia, 65:324-337.

17. Hongo, S,; Nakashima, Y,; Akomo-Okoue, Ef,; Mindonga Nguelet, Fl. (2016). Female Reproductive Seasonality and Male Influxes in Wild Mandrills (Mandrillus sphinx). International Journal of Primatology. 37(3) : 416-437

18. IWATA, Y,; ANDO, C. (2007). Bed and bed-site reuse by western lowland gorillas (Gorilla g. gorilla) in Moukalaba-Doudou National Park, Gabon. Primates, vol. 48: 77-80.

19. KIERULFF, MC,; SANTOS, GR,; CANALE, GR,; GUIDORIZZI, CE,; CASSANO, CR,; 2004. The use of camera-traps in a survey of the buff-headed capuchin monkey, Cebus xanthosternos. Neotrop. Primates, 12: 56-59.

20. KINGDON, J,; HAPPOLD, D,; BUTYNSKI, T,; HOFFMANN, M,; HAPPOLD, M,; KALINA J. (2013). Mammals of Africa: Bloomsbury Publishing, London Volumes 1-6.

21. MAMBEYA, MM,; BAKER, F,; MOMBOUA, BR,; KOUMBA PAMBO, AF,; HEGA, M,; OKOUYI OKOUYI, VJ,; ONANGA, M, CHALLENDER, DWS,; INGRAM, DJ,; WANG, H ABERNETHY $K$, (2018). The emergence of a commercial trade in pangolins from Gabon. African Journal of Ecology; 00:1-9. https://doi.org/10.1111/aje.12507.

22. MANGAMA-KOUMBA, LB.; NAKASHIMA, Y.; MAVOUNGOU, JF.; AKOMO-OKOUE, EF.; YUMOTO, T,; YAMAGIWA, J,; M'BATCHI, B. (2016). Estimating diurnal primate densities using distance sampling method in Moukalaba-Doudou National Park, Gabon. Journal of Applied Biosciences. 99: 9395 - 9404. 
23. MINDONGA NGUELET, FL; ZINGA-KOUMBA, CR,; MAVOUNGOU, JF,; NZENGUE, E, AKOMO-OKOUE, EF,; NAKASHIMA, Y,; HONGO, S,; EBANG-ELLA, GW; MANGAMA-KOUMBA, LB,; M’BATCHI, B. (2016). Etude de la relation entre l'abondance des grands mammifères frugivores et celle des fruits dans le Parc National de Moukalaba-Doudou, Gabon. International Journal of Biological and Chemical Sciences, 10(5): 1969-1982.

24. MOUNIOKO, F., ZINGA KOUMBA C.R., MBANG NGUEMA O.A., DIBAKOU E.S., ACAPOVI-YAO, G., M'BATCHI, B., MAVOUNGOU, J.F. (2016). Eco-distribution des mouches piqueuses, vecteurs de la Trypanosomose humaine africaine, dans le Parc National de Moukalaba Doudou (Sud-Ouest du Gabon) en saison sèche. ElWahat pour les Recherches et les Etudes, 9 (1) : 50-64.

25. NAKASHIMA, Y,; INOUE, E,; AKOMO EO. (2013a). Population density and habitat preference of forest duikers in the MoukalabaDoudou National Park, Gabon. African Zoology, 48(2): 195-399.

26. NAKASHIMA, Y,; IWATA, Y,; ANDO, C,; NKOGUE, CN,; INOUE, E,; AKOMO, EO,; NGUEMA, PM,; BINENI, TG,; BANAK, LN,; TAKENOSHITA, Y,; NGOMANDA, A,; YAMAGIWA J. (2013b). Assessment of landscape-scale distribution of sympatric great apes in African rain $\neg$ forests: Concurrent use of nest and camera-trap surveys. American Journal of Primatology, 75: 1220-1230.

27. NAKASHIMA, Y. (2015). Inventorying medium- and large-sized mammals in the African lowland rainforest using camera trapping. Tropics, 23(4): 151-164.

28. NELSON, A,; BIDWELL, P. AND SILLERO-ZUBIRI, C. (2003). A review of human-elephant conflict management strategies. People and Wildlife Initiative. Wildlife Conservation Research Unit, Oxford University press.

29. O'BRIEN, TG,; KINNAIRD, MF,; WIBISONO, HT. (2003). Crouching tigers, hidden prey: Sumatran tiger and prey populations in a tropical forest landscape. Animal Conservation, vol. 6(2): 131-139.

30. PEBSWORTH, P,; LAFLEUR, M. (2014). Advancing primate research and conservation through the use of camera traps: introduction to the special issue. International Journal of Primatology., 34(5): 825-840. DOI: 10.1007/s10764-014-9802-4.

31. RIEUCAU J. (2001). Biodiversité et écotourisme dans les pays du centre du golfe de Guinée Prétourisme dans une unité géopolitique instable, Les Cahiers d'Outre-Mer, 216: 417-452. 
32. ROGER, A,; CALAQUE, R,; DOUMENGE, C. (2006). Une évaluation du potentiel écotouristique du parc national des plateaux Batéké. Bois et Forêts des Tropiques, 290(4):13-30.

33. TAKENOSHITA, Y,; ANDO, C,; IWATA, Y,; YAMAGIWA, J, (2008). Fruit phenology of the great habitat in the Moukalaba-Doudou National Park, Gabon. African. Study. Monograph, 39S:23-39.

34. TAKENOSHITA, Y,; ANDO, C,; YAMAGIWA J. (2008). Fruit phenology of the great ape habitat in the Moukalaba-Doudou National Park, Gabon. African Study Monographs, 39: 23-39.

35. TOBLER, MW,; CARRILLO-PERCASTEGUI, SE,; LEITE PITMAN, R,; MARES, R,; POWELL, G. (2008). An evaluation of camera traps for inventory-ing large- and medium-sized terrestrial rainforest mammals. Animal Conservation, 11(3): 169-178.

36. TROLLIET, F,; HUYNEN, M-C; VERMEULEN, C; HAMBUCKERS, A. (2014). Use of camera traps for wildlife studies. A review. Biotechnological. Agronomic Social. Environement., 18(3) : 446-454.

37. TUTIN, CEG,; FERNANDEZ, M. (1993). Relationships between minimum temperature and fruit production in some tropical forest trees in Gabon. Journal of Tropical Ecology, 9:241-248.

38. TUTIN, CEG,; WHITE, L,; MACKANGA-MISSANDZOU A. 1997. The use by rain forest mammals of natural forest fragments in an equato $\neg$ rial African savanna. Conservation Biology, 11(5): 11901203.

39. TUTIN, CEG,; FERNANDEZ, M .1984. Nationwide census of gorilla (Gorilla g. gorilla) and chimpanzee (Pan t. troglodytes) populations in Gabon. American Journal of Primatology, 6: 313-336.

40. VANDE, W.J.P. (2012). MOUKALABA DOUDOU. Agence National des Parcs Nationaux (ANPN), Libreville, Gabon. 296 p.

41. WHITE, LJT. (1994). Biomass of rain forest mammals in the Lopé Reserve, Gabon. Journal of Animal Ecology, 63: 499-512.

42. WHITE, LJT,; EDWARDS, A. (2000). Conservation research in the African rain forests: a technical handbook. The Wildlife Conservation Society, New York.

43. YAMAGIWA, J,; BASABOSE, AK. (2006). Diet and seasonal changes in sympatric gorillas and chimpanzees at Kahuzi-Biega National Park. Primates DOI 10.1007/s10329-005-0147-7. 47: 74-90 